\title{
A Novel Method for 3D Image Segmentation with Fusion of Two Images using Color K-means Algorithm
}

\author{
Neelam Kushwah \\ Dept. of CSE \\ ITM Universe \\ Gwalior
}

\author{
Priusha Narwariya \\ Dept. of CSE \\ ITM Universe \\ Gwalior
}

\begin{abstract}
In this proposed paper presented $3 \mathrm{D}$ image segmentation and fusion of two images using color automatic k-means clustering algorithm. It is a low level operation concerned with separating of images using calculating similarity or discontinuity, or homogeneously, by finding edges or boundaries'. Image segmentation is the process of splitting an image into several partitions, so as to change the optimization of an image into somewhat that is more expressive and easier to analyze. The experimental results give better results in terms of normalized cross correlation, absolute error and execution time. It gives improved results as compared to level set segmentation method.
\end{abstract}

\section{Keywords}

Image Segmentation; K-means; Fusion; level set.

\section{INTRODUCTION}

Imaging methodologies in Medicine prepare the doctors to see the portions of the interior of the body for the purpose of more easy diagnosis. Analysis on computer-based facial expression and effect analysis has deepened since the first FG session in 1995. The resulting advances have made the emerging field of affective computing possible. The continued improvement of emotion-capable systems significantly depend on access to well-annotated, illustrative affecting corpora [6]. A amount of the $2 \mathrm{D}$ facial expression databases have become presented (e.g., $[1,2,3,4,9])$, as well as certain with $3 \mathrm{D}$ imaging data (e.g., [5,7,8,12,13,15]). Although particular systems have been effective, presentation degrades when handling expressions with low intensity appearance, large head rotation, subtle skin movement, and/or lighting variations by changing postures. Because of the limits of defining facial surface deformation when $3 \mathrm{D}$ features are estimated in $2 \mathrm{D}$ images, 2D space with a handful of features may not reflect exactly the authentic facial expressions (e.g., in-depth motion of the 3D wrinkles, 3D head pose and skin extrusion in the areas of the crow's feet,cheek, forehead, glabella, and nasolabial). Another problematic subject is that facial AUs (action units) can happen in more than 7000 different complex mixtures [10], causing bulges and various in- and out-ofimage-plane movements of permanent facial features, negative emotions from vision of the left hemi face, and mouth extrusions that are challenging to $2 \mathrm{D}$ plane detect. analysis and tracking of Three-dimensional dynamic surface will be significant for those facial expressions for which 2D motion knowledge is not enough. Because the face is a 3D object and numerous communicative signals involve changes in depth and head rotation, inclusion of 3D information is an important addition. Another major limitation of existing databases is that most have only acted facial behavior or posed, and thus the document are not representative of spontaneous affecting expression, which may change in intensity, timing, and complexity of posed expression [11]. No presently existing dataset holds dense, dynamic, 3D facial representations of the spontaneous FACS (facial expression with anatomically based) annotation [14].

Fast growth in the multimedia and its associated fields extremely growths the size of the image sources in various fields such as entertainment, medicine, media, commerce, and engineering. Concurrently, the significant growth in the use of images for research, training and education in divherse fields significantly demands for an efficient and effective method for retrieving and storing the images in/from the vast repositories, which is very problematic and most challenging jobs than ever before for a study community. The classical image retrieval methods rely on the manually annotated textual keywords [16]. With the huge-scale image repositories, the outcomes of textual keyword based on the retrieval systems are not fully dependable because of the usage of the limited number of textual keywords, being tedious, subjective, laborious and time-consuming. The CBIR (content based image retrieval) system disables the shortcomings of textual keyword based image retrieval systems and it is an efficient and effective result to deal with the huge-scale image repositories. Thus, many in effect content based image retrieval systems have been presented in the literature [17-25] since 1990s with varying degree of automation, reliability and capability. The images in the content based image retrieval systems are represented by a collection of visual contents such as shape, color, texture, and spatial.

Clustering is a common technique by which an image is divided into multiple segments. Clustering method can be measured the most significant unsupervised learning difficulty, which deals with detecting a structure in a set of unlabeled data. K-means clustering and FCM (fuzz c-means) clustering are two most important unsupervised algorithms. The K-means algorithm, which is the computationally hard (NP-hard), is an iterative method that is used to divide an image into $\mathrm{K}$ clusters [36]. A method was originally explain by Jim Bezdek in 1981 a common data clustering method in FCM is a in which all facts position which is belongs to a cluster to little degree which is explain with the help of a membership grade. [37] As a development on previous clustering techniques. FCM algorithm assimilates spatial knowledge in the clustering for membership function. The spatial meaning is the summing up of membership function in the neighborhood of all pixels in consideration [38].

\section{LITERATURE REVIEW}

Xing Zhang (2014), central of human experience is Facial expression. The main issue is efficiency and valid measurement that automated facial image inquiry seeks to the location. The posed and un-posed facial expressions are 
different along several dimensions in which including timing and including complexity.

Hongbing Liu (2014), Color image segmentation algorithms are suggested based on the GrCC (granular computing clustering). Firstly, the atomic hyper-spherical granule is signified as the vector containing the RGB value of pixel of color images and radii 0 . Next, the union operator of two hyper-spherical granules is designed to obtain the larger hyper-spherical granule compared with these two hyperspherical granules. Thirdly, the granular computing clustering is established by the union operator and also the user-defined granularity threshold. GCE (Global Consistency Error), VI (Variation of Information), RI (Rand Index), and $\Delta$ En (Loss Entropy) are used to assess the segmentations. color images Segmentations selected from BSD300 and internet show that segmentations by granular computing clustering speed up the segmentation procedure and attain the better segmentation presentation associated with K-means and FCM segmentations

Haida Liang (2014), Portable Remote Imaging System for Multispectral Scanning (PRISMS) is creating for in situ 3D topographic imaging of wall paintings and, high resolution spectral and another huge plot. In generally, it may figure at slanting resolutions of tens of microns distantly from distances of tens of meters, creating a high motion imaging which is probable from an unchanging position for areas at heights that is confront to access. A fully automated spectral imaging system giving 3D topographic mapping at millimeter correctness as a consequence of the image focusing process.

AlardRoebroeck (2014), Multimodal neuro-imaging has turn into a foundation of essential and cognitive neuro-science in humans and animals, even though challenges to judge when acquiring and joining non-redundant imaging data. In this survey, it discussed data acquisition and fusion in multimodal neuro-imaging in the framework of all of these potential merits. However, due to various limitations in the neuronal and structural under-pinning's of all method - have to be occupied into the account when interpreting and modeling multimodal data by generative models.

C. Bhuvaneswari (2014), Automatic classification of lung diseases in CT (computed tomography) images is a dynamic analytic tool for the computer-aided diagnosis system. In this study, it proposed a novel image based feature removal method for classification of lung computed tomography images. A new fusion based on technique was established by merging the Walsh Hadamard transform features and Gabor filter applying MAD (median absolute deviation) method and hence, it holds the benefits of both models. The suggested system includes of three phases. In the first phase, the images are features and preprocessed are removed by new fusion based feature extraction method, followed by second phase, in which extracted features are selected with the help of genetic algorithm which selects top ranked features. In the last stage, classifiers namely decision tree, KNN (K nearest neighbor), MLP-NN (Multi layer perceptron Neural Networks) are employed to achieve classificationof lung diseases. A total of the 400 datasets for the pleural effusion, diseases bronchitis, emphysema and normal lung were used for testing and training. The system has been tested with aamount of real Computed Tomography lung images and has attained suitable outcomes in categorizing the lung diseases.

Salah-Eddine Lamhamedi-Cherradi (2014), Despite longstanding reliance upon monolayer culture no. of benefit from both a practical and experimental for studying cancer cells, and standpoint, a complex problem three dimensional (3D) models of growing body of evidence suggests that much are impotent to properly take off many of the serious hallmarks connected with the on cogenesis, maintenance and spread of ES (Ewing's sarcoma), the second most generally pediatric bone tumor.

Jeroen De Reu (2014)- It described the possibilities and restrictions of image-based 3D modeling in the video recording of an entire mine. It suggested the idea that imagebased 3D modeling can be a brilliant and appropriate knowledge for the video recording, credentials and visualization. It suggestions great options for growing the feature of the archived archaeological excavation record. They believe that image-based 3-dimensional modeling can cause development in archaeological mine practice.

Feng Zhao (2013), in this paper, He presents that an overview on medical images based interactive segmentation technique. Region-based and Edge-based level set segmentation technique provides a direct way to estimate the geometric properties of anatomical structures. They are popular as a general framework for many applications of medical image analysis, such as brain MRI images and 3D CT of carotid arteries.

ThordAndersson (2013), Level set technique are a popular way to resolve the image segmentation issue. In a series of 2D or 3-D-images by means of real and artificial data with position fact, the modifications are exposed to decrease the sensitivity for narrow optima and to enlarge the convergence speed. The experimental result contour is start by solving an optimization difficulty where a cost functional is minimized, however, perceptive to local minima and often show slow junction. In the previous method, cost functional has been customized to evade these problems. In this paper, they instead propose by means of two modified gradient descent methods, using a momentum term and one based on resilient propagation.

Bing Luo (2012), Creep drift of piezoelectric actuators in the solder paste 3D inspection was hard to be correct standardized. Associated with actuated projecting rough, this paper suggested a novel calibration method that a quartercycle sinusoidal rough was used for estimate to substitute usual cycle grating and also two phases-shift sinusoidal gray images were matched to displacements calculate. As calibration situations containing image capturing time delay were the similar as in 3D measuring occupied, the non-linear of PZT and creep drift were all measured. Experimental outcomes display that the PZT was 3D reconstructed and delicately calibrated efficiently accuracy was enhanced.

Indah Soesanti (2011), it proposed optimized fuzzy logic segmentation for abnormal MRI brain images analysis is presented. Spatial knowledge in the image does not use by a conventional fuzzy c-means (FCM) method. In this analysis, a FCM algorithm that incorporates spatial knowledge into membership function for the clustering.

\section{PROPOSED METHODOLOGY}

There is a need of medical integration because there is too many data, data dependency and physical requirements. The goal of medical integration is being achieved through the process of the Registration, segmentation, fusion techniques. Following are the methods used: 


\subsection{Registration}

Image registration is done at several view-points either $2 \mathrm{D}$ or 3 -D. there are number of algorithms for registration of images like area based registration, feature based.

\section{a) Select Original Image}

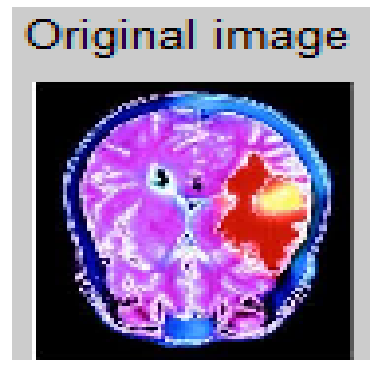

Fig 1: Take an original image

\subsection{Segmentation}

For most medical image analysis tasks the Segmentation of Image is a most necessary method. Having good segmentations will benefit clinicians and patients as they provide necessary knowledge for the purpose of early disease detection, surgical planning and 3-D visualization.

\section{b) Segmented Image}

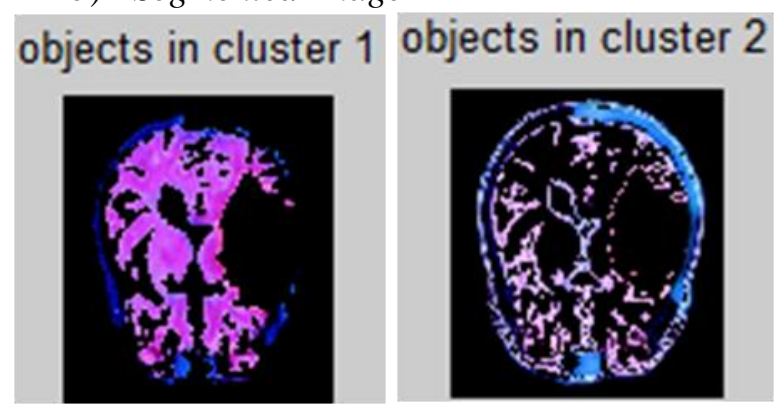

(a)

(b)

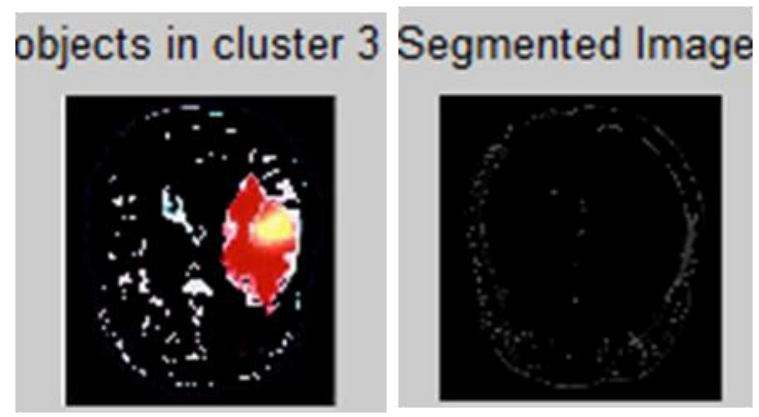

(c)

(d)

Fig 2: No. of Cluster Images

\subsection{Fusion}

In the fusion process, it merges two images like mri, ct scan images. In the Fast Discrete Transform by Wrapper algorithm based image fusion technique.

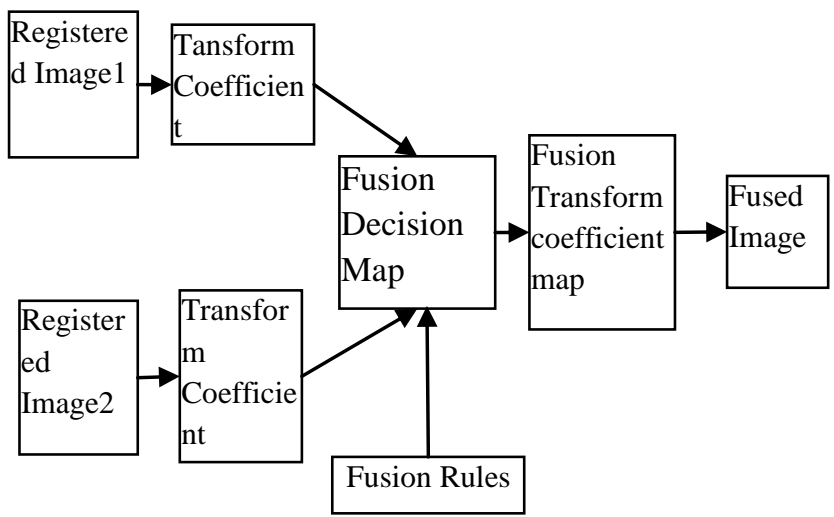

Fig 3: Structure of Fusion System

\subsection{Proposed Algorithm}

1. Take RGB color image is to be converted into $\mathrm{L} * \mathrm{a} * \mathrm{~b} *$ color space.

2. The $\mathrm{L}^{*} \mathrm{a} * \mathrm{~b} *$ break the brightness layer into ' $\mathrm{L} *$ ', chromaticity-layer ' $\mathrm{a}^{*}$ ' $=$ the length of the red-green axis, and ' $b{ }^{* '}=$ the length of the blue-yellow axis

3. Perform k-means clustering algorithm on ' $a * b$ ' for classification.

4. Repeat the clustering steps 3 times to avoid local minima.

5. Perform k-means to collect the objects into three clusters on the basis of Euclidean distance.

6. Obtain segmented image using Color.

7. Calculate mean values of every cluster_index.

8. Apply fusion algorithm for merge two images using this

9. Calculate normalized cross-correlation between original and segmented image.

$\mathrm{NK}=\frac{\text { sum(sum(O_imgXSeg_img }))}{\operatorname{sum}\left(\operatorname{sum}\left(O_{-} i m g X O_{-} i m g\right)\right)}$

Where NK is normalized cross-correlation, $\mathrm{O}$ img is original image and Seg_img is segmented image.

10. Calculate normalized absolute error difference between original and segmented image.

$\mathrm{NAE}=\frac{\operatorname{sum}(\operatorname{sum}(\text { abs }(\text { error })))}{\operatorname{sum}\left(\operatorname{sum}\left(O_{-} \text {img }\right)\right)}$

Where MXN is size of the image, $\mathrm{AD}$ is average difference

11. Calculate mean square error between original and segmented image.

$\mathrm{MSE}=\frac{\text { error }+ \text { double }\left(\left(O \_i m g-S e g \_i m g\right)^{\wedge} 2\right)}{M X N}$ 


\section{RESULT ANALYSIS}

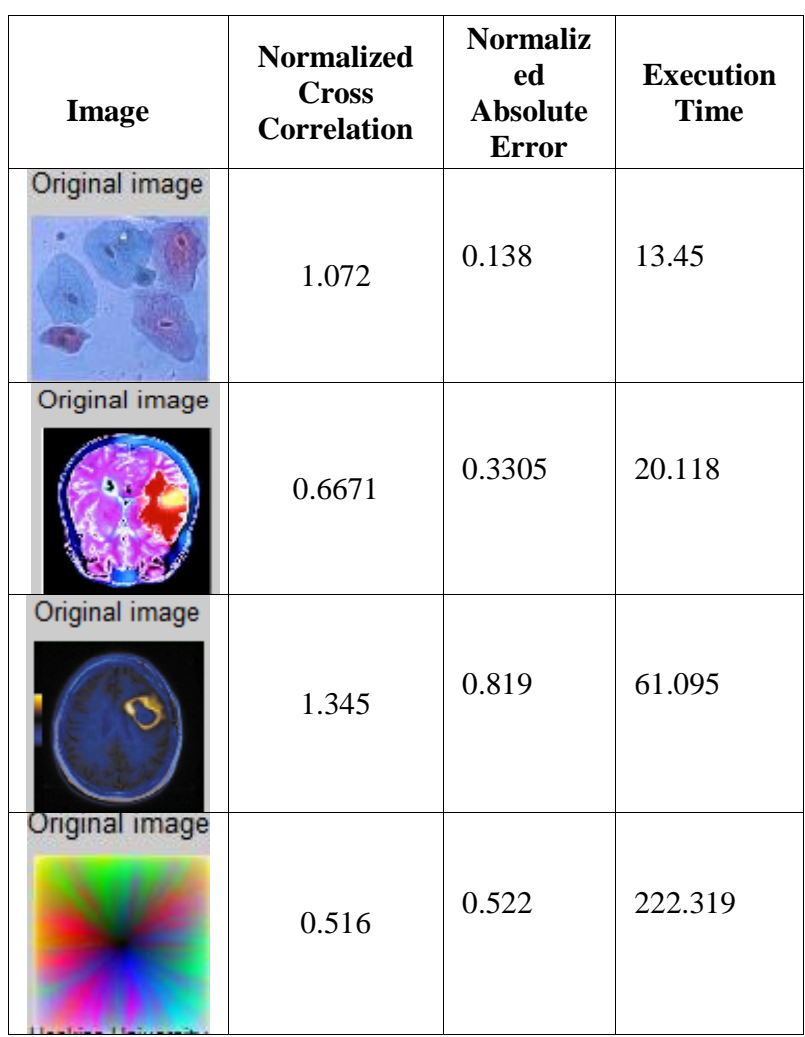

Table1. For Base System

\begin{tabular}{|c|c|c|c|}
\hline Image & $\begin{array}{c}\text { Normalized } \\
\text { Cross } \\
\text { Correlation }\end{array}$ & $\begin{array}{c}\text { Normali } \\
\text { zed } \\
\text { Absolute } \\
\text { Error }\end{array}$ & $\begin{array}{c}\text { Execution } \\
\text { Time }\end{array}$ \\
\hline Original image & 0.079 & 0.906 & 1.93 \\
\hline Original image & & 0.9766 & 1.7180 \\
\hline Original image & & & \\
\hline Original image & & 0.0082 & \\
\hline
\end{tabular}

Table 2. For Proposed System

\subsection{Graph Analysis}

Normalized Cross Correlation- The time delay between two signals is determined using the argument of the maximum. For image-processing applications in which the intensity of the picture and model can vary due to lighting and exposure circumstances, the images can be first normalized.

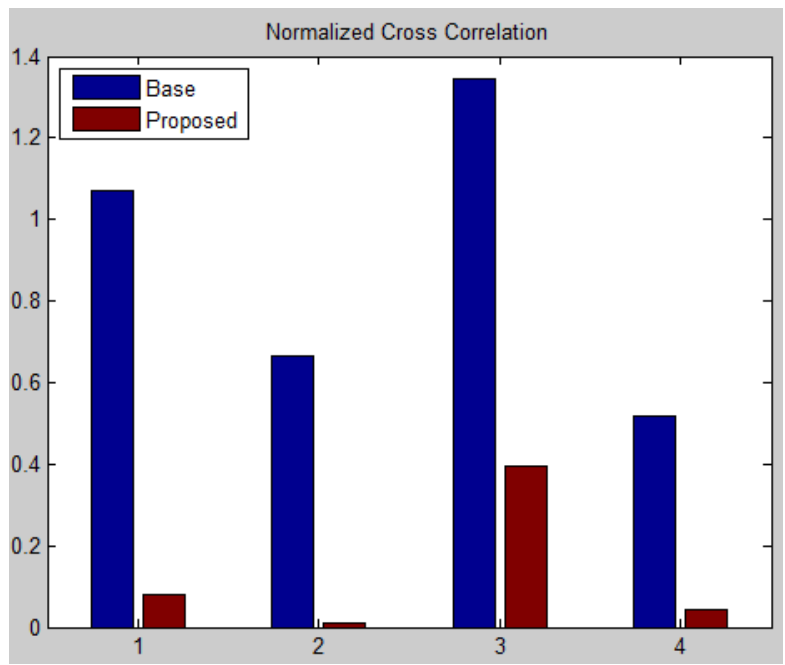

Graph 1: Comparison between base and proposed in Normalized Cross Correlation

In graph 1 shows the comparison between base technique and proposed technique in terms of NCC. The intensity of image in proposed work is better compare to other.

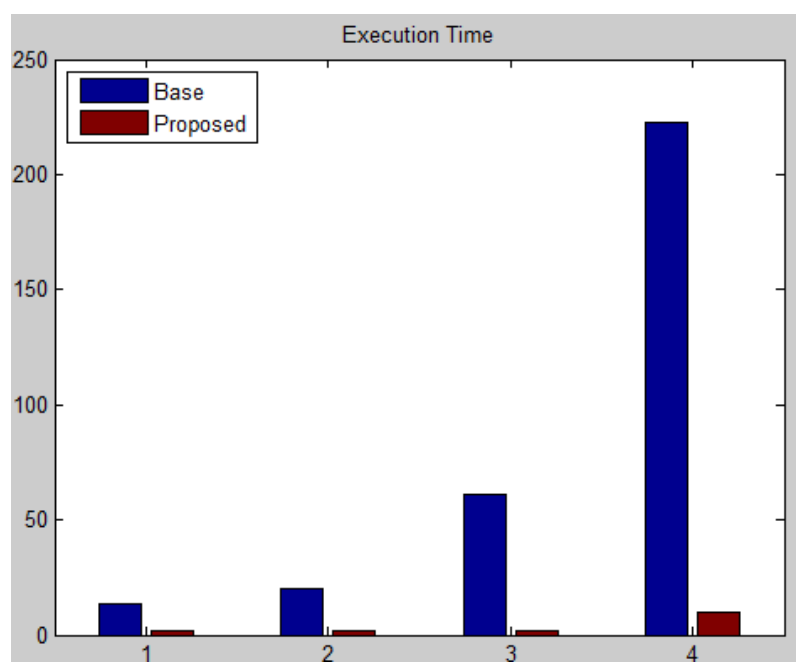

Graph 2: Comparison between base and proposed Execution time

In Graph 2 shows the execution time of proposed work which is better from previous work. 


\section{CONCLUSION}

In this paper proposed a novel approach of 3D image segmentation on color image using K-means clustering method. Time, normalized absolute error and normalized cross correlation have been the main focus here. But there are still restrictions that like k-means segmenting with prearranged number of clusters. The calculated properties showed that the results of the K-Means and level set method are much closer to each other than the Segmentation method. However, the results demonstrated that these techniques can be effectively used to find and extract the brain tumor in MRI images and also nuclei images, which can be included with the outcome of other studies to facilitate doctors in surgery, diagnosis, treatment planning and monitoring. In future work also apply machine learning algorithms like SVM and neural networks for image segmentation.

\section{REFERENCES}

[1] T. Kanade, J.F. Cohn, Y. Tian, Comprehensive Database for Facial Expression Analysis, IEEE International Conference on Automatic Face and Gesture Recognition (FG), France, 2000.

[2] E. Douglas-Cowie, R. Cowie, et al., The Humaine Database: Addressing the Collection and Annotation of Naturalistic and Induced Emotional Data, ACII, 2007, pp. 488-500.

[3] T. Anziger, K. Scherer, Using Actor Portrayals to Systematically Study Multimodal Emotion Expression: The GEMEP Corpus, ACII, 2007.
[4] A. Savran, N. Alyuz, et al., Bosphorus Database for 3D Face Analysis, BIOID, 2008, pp. 47-56.

[5] Z. Zeng, M. Pantic, G. Roisman, T. Huang, A survey of affect recognition methods: audio, visual, and spontaneous expressions, IEEE Trans. Pattern Anal. Mach. Intell. 31 (1) (2009) 39-58.

[6] L. Yin, X.Wei, Y. Sun,W. J., M. Rosato, A 3D Facial Expression Database for Facial Behavior Research, IEEE Inter. Conf. on Automatic Face and Gesture Recognition, Southampton, UK, 2006, 2006.

[7] L. Yin, X. Chen, Y. Sun, T. Worm, M. Reale, A Highresolution 3D Dynamic Facial Expression Database, IEEE Inter. Conf. on Automatic Face and Gesture Recognition, Amsterdam, the Netherlands, Sept. 2008, Sept. 2008

[8] P. Lucey, J.F. Cohn, J. Saragih, I. Matthews, Z Ambadar, The Extended Cohn-Kanade Database: A Complete Facial Expression Database for Both Facial Action Units and Emotion Detection, IEEE CVPR4HB, 2010 .

[9] Ajala Funmilola A*, Oke O.A, Adedeji T.O, Alade O.M, Adewusi E.A Department, "Fuzzy k-c-means Clustering Algorithm for Medical Image Segmentation", Vol 2, No.6, 2012

[10] Rabab Saadoon Abdoon, "Clustering and Enhancement Methods for Extracting 3D Brain Tumor of MRI Images", Volume 3, Issue 9, September 2013. 